Document downloaded from:

http://hdl.handle.net/10251/50136

This paper must be cited as:

Cantó Colomina, B.; Coll, C.; Sánchez, E. (2014). Structured parametric epidemic models. International Journal of Computer Mathematics. 91(2):188-197. doi:10.1080/00207160.2013.800864.

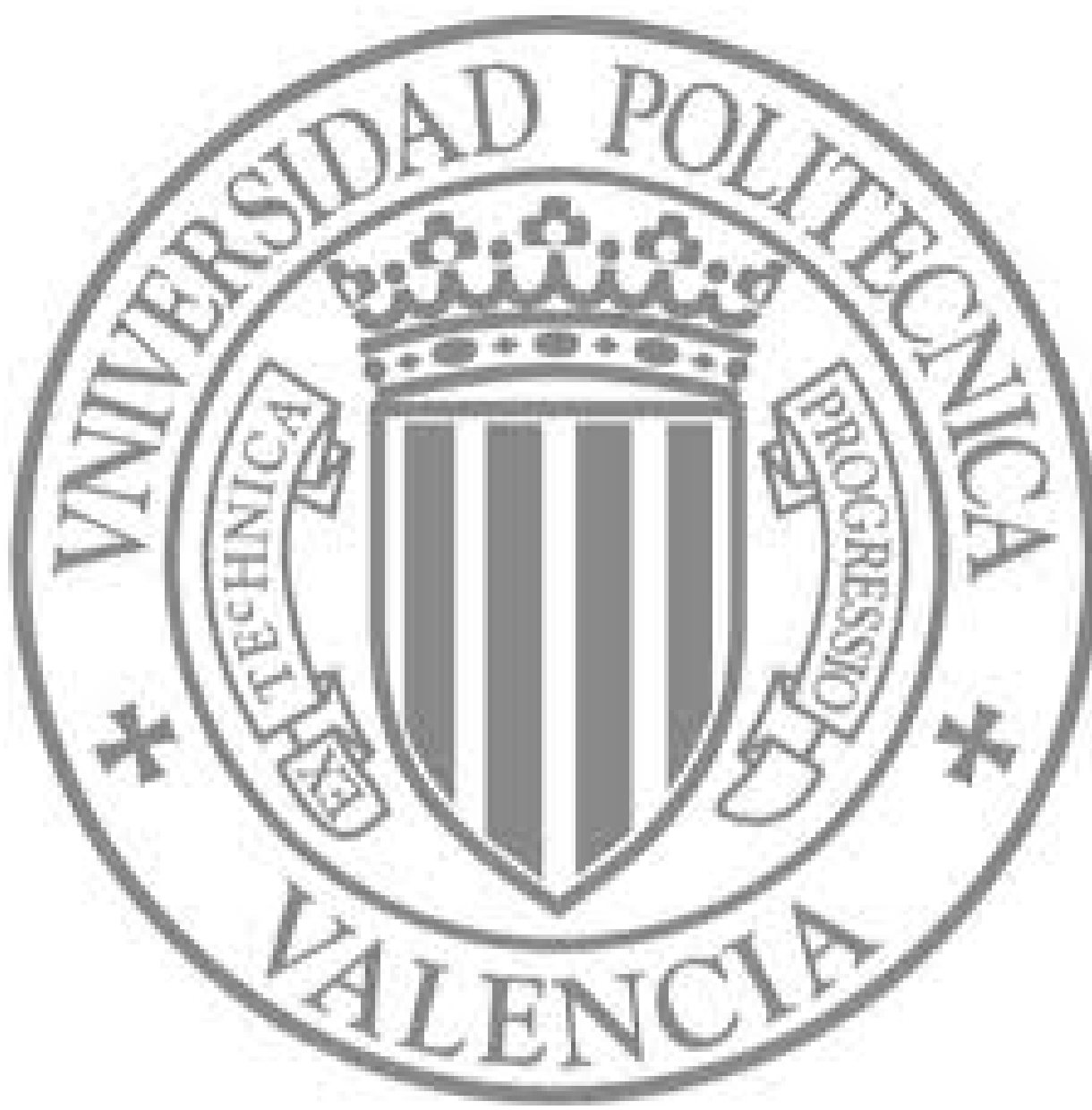

The final publication is available at

http://dx.doi.org/10.1080/00207160.2013.800864

Copyright Taylor \& Francis: STM, Behavioural Science and Public Health Titles 


\title{
Structured parametric epidemic models
}

\author{
B. Cantó** C. Coll and E. Sánchez \\ 4 Instituto de Matemática Multidisciplinar, Universitat Politècnica de València, Camino de Vera
}

May 12, 2015

\begin{abstract}
A stage-structured model for a theoretical epidemic process that incorporates immature, susceptible and infectious individual in independent stages is formulated. In this analysis, an input interpreted as a birth function is considered. The structural identifiability is studied using the Markov parameters. Then, the unknown parameters are uniquely determined by the output structure corresponding to an observation of infection. Two different birth functions are considered: the linear case and the Beverton-Holt type to analyze the structured epidemic model. Some conditions on the parameters to obtain nonzero diseasefree equilibrium points are given. The identifiability of the parameters allows us determine uniquely the basic reproduction number $\mathcal{R}_{0}$ and the stability of the model in the disease-free equilibrium is studied using $\mathcal{R}_{0}$ in terms of the model parameters.
\end{abstract}

\section{Introduction}

For an epidemic model, one of the most important parameters in the study of mathematical epidemiology is the basic reproduction number. That is, to find the number of new infections produced by a typical infective individual in a population in a disease-free equilibrium. The basic reproduction number can be used to assess whether a newly infectious disease can invade a population [14]. Some results on discrete epidemic models are given in [1] and [9] and continuous models have been studied by [16]. The interest of this number lies in when the basic reproduction number is less than one the disease-free equilibrium is asymptotically stable and when the basic reproduction number is greater than one it is unstable [7].

On the other hand, in an epidemic model there are several unknown parameters. The determination of these parameters is critical for the design or control of epidemic models. For that, the study of the identifiability analysis is important. The problem of the structural identifiability of the model consists of the determination of all parameter sets which give the same input-output structure. A characterization of structural identifiability is given in [3]. Thus, a system is structurally identifiable if and only if there is an unique input-output behavior for every parameter set. One characteristic of this property is that the parameters have physical significance and it is possible to determine their values from observed data.

For a mathematical epidemic model, a discrete-time approach is more realistic than differential one, since the epidemic statistics are made from given time intervals and not continuously (a fact that may be important for the rapid spread of epidemics). In particular, such systems are very useful in the case of

\footnotetext{
**Corresponding author. Email: bcanto@mat.upv.es
} 
age-dependent and time-dependent models of infectious diseases where such information has to be part of the model. Examples of discrete epidemic models can be found in [2, 6] and references given there.

The aim of this work is to obtain a theoretical model to analyze a hypothetical epidemic. We consider SIS type of disease transmission with unknown parameters, since we suppose the individuals recover with no immunity to the disease. The unknown parameters depend on the considered epidemic and it is important to ensure that these parameters can be determined from experimental data. For that, we study the identifiability of the model. Moreover, we find the equilibrium of the system and we analyze the stability in the disease-free equilibrium point using the basic reproduction number.

We propose a discrete-time epidemic model with the disease that spreads only among mature individuals. The population is subdivided, according to the disease state, into three classes:

- immature/juvenile or immune individuals, that is, individuals who cannot be infected. From now on, we will write it simply inmature and denote it by $j$;

- susceptible mature individuals, that is, individuals may become infected. From now on, we will write it simply susceptible and denote it by $s$.

- infectious mature individuals. From now on, we will write it simply infectious and denote it by $i$.

In addition, the host population is subdivided into two classes, male and female, denoted as $m$, and $f$, respectively. That is, let $j_{m}(t), j_{f}(t), s_{m}(t), s_{f}(t), i_{m}(t)$ and $i_{f}(t)$ be the density/percentage of immature, susceptible and infectious, males and females, respectively.

Before describe the model it is assumed that the susceptible individual become infectious after contact with infective individuals and recovery from disease does not give permanent immunity. Furthermore, we suppose that only have ability of breeding the susceptible female. This assumption is acceptable. For example, some sexually transmitted diseases like gonorrhea where infected women cannot give birth to babies.

On parameters: let $k_{1}$ be the survival rate of the immature individual, $k_{2}$ is the survival rate of the susceptible individual and $k_{3}$ is the survival rate of the infected individual, $c$ is the probability of immature individual becoming susceptible individual, $g$ is the probability that susceptible individual become infectious individual and $\sigma$ is the probability of infected individual recover. On the other hand, $b(t)$ is the birth number of the male and female individuals, respectively.

These assumptions lead to the following discrete population model with stage structure,

$$
\begin{aligned}
j_{m}(t+1) & =k_{1}(1-c) j_{m}(t)+b(t) \\
j_{f}(t+1) & =k_{1}(1-c) j_{f}(t)+b(t) \\
s_{m}(t+1) & =k_{1} c j_{m}(t)+k_{2}(1-g) s_{m}(t)+k_{3} \sigma i_{m}(t) \\
s_{f}(t+1) & =k_{1} c j_{f}(t)+k_{2}(1-g) s_{f}(t)+k_{3} \sigma i_{f}(t) \\
i_{m}(t+1) & =k_{2} g s_{m}(t)+k_{3}(1-\sigma) i_{m}(t) \\
i_{f}(t+1) & =k_{2} g s_{f}(t)+k_{3}(1-\sigma) i_{f}(t)
\end{aligned}
$$

The proposed model can be used to predict and control many diseases such as hantavirus, herpes zoster or gonorrhea. Many diseases only spread among mature individuals and the stage-structure model is useful, see [1, 6, 13]. The time intervals depend on the specific disease and the type of the population. Normally, the time interval $[t, t+1]$ is approximately the gestation period plus the time until sexual maturity. 
To analyze an epidemic model worth knowing how the population increases. Since only the susceptible female have ability of breeding, we consider two forms for $b(t)$. The first one is a linear birth function given by

$$
b(t)=\alpha s_{f}(t)
$$

A good reason to use linear functions is their simplicity in describing the processes allowing the discussion of the assumptions made in it and the conclusions drawn from the biological point of view. Note that, the linear recruitment function can be described simply in terms of their reproduction. However, this kind of functions does not usually reflect the real recruitment processes.

The second is a Beverton-Holt type used for example in [1] and [13]. If $n(t)=j_{m}(t)+j_{f}(t)+s_{m}(t)+$ $s_{f}(t)+i_{m}(t)+i_{f}(t)$, the function is given by

$$
b(t)=\frac{\alpha s_{f}(t)}{1+\beta n(t)}
$$

where $\alpha>1$ and $\beta>0$ are constant. Usually, $\frac{1}{1+\beta n(t)}$ can be interpreted as the probability of survival till next year. The discrete logistic equation as the Beverton-Holt type contains self-regulatory mechanisms that are density dependent, that is, the growth depends nontrivially on the current population. Note that, the Beverton-Holt type recruitment function represents a non-linear relationship between the total population and the recruitment. That is, when the population level is low, the recruitment increases as population increase, when the population exceeds a certain level, the recruitment becomes saturated as the population increase. This kind of function has the advantage that best describes the mechanism arising naturally from simpler models of epidemic processes but it has disadvantage that can be complicated to analyze.

Therefore it is important to establish that nonnegative initial data give rise to nonnegative solution. In our model this property is guaranteed by the non-negativity of the coefficient matrices of the system (see [10]).

The organization of this paper is the following. In Section 2, the identifiability problem is studied. In Section 3, the equilibrium point of the model is obtained, developed for the study of two cases given by (7) and (8). In Section 4, a reproductive number is studied for a compartmental discrete epidemic model, using for $b(t)$ a linear function and finally we obtain this number using a Beverton-Holt type function.

\section{Identifiability}

An important prerequisite to interpreting results from parameter estimation and inferring characteristics of a real system is the structural identifiability analysis. It is an important step in the modeling process and it is necessary theoretical prerequisites to design the experiment and to identify the system. For that, it is possible estimate the unknown parameters of the model using experimental data (more information in [5]). Structural identifiability guarantees that the model parameters can be estimated uniquely, under ideal conditions. If the system is identifiable it is possible to find an state-space model of the system whose input-output response is the same as of an input-output parameterized model.

Considering the representation of Eqs. (1.6), we have the following system

$$
x(t+1)=A(\mathbf{p}) x(t)+B b(t)
$$

where

$$
x(\cdot)=\left(j_{m}(\cdot) j_{f}(\cdot) s_{m}(\cdot) s_{f}(\cdot) i_{m}(\cdot) i_{f}(\cdot)\right)^{T}
$$




$$
\begin{aligned}
A(\mathbf{p}) & =\left(\begin{array}{cccccc}
k_{1}(1-c) & 0 & 0 & 0 & 0 & 0 \\
0 & k_{1}(1-c) & 0 & 0 & 0 & 0 \\
k_{1} c & 0 & k_{2}(1-g) & 0 & k_{3} \sigma & 0 \\
0 & k_{1} c & 0 & k_{2}(1-g) & 0 & k_{3} \sigma \\
0 & 0 & k_{2} g & 0 & k_{3}(1-\sigma) & 0 \\
0 & 0 & 0 & k_{2} g & 0 & k_{3}(1-\sigma)
\end{array}\right) \\
B & =\left(\begin{array}{llllll}
1 & 1 & 0 & 0 & 0 & 0
\end{array}\right)^{T}
\end{aligned}
$$

and the parameter vector $\mathbf{p}$, which belongs to a subset of admissible parameters $\mathcal{P} \subseteq \mathbb{R}^{r}$, is given by the parameters $c, g$ and $\sigma, \mathbf{p}=(c, g, \sigma)$. If the matrices $A(\mathbf{p})$ and $B$ are nonnegative, then solutions corresponding to nonnegative initial data remain nonnegative in the future (see [10]). The nonnegative condition on $A(\mathbf{p})$ means that the parameters satisfy $c \leq 1, g \leq 1$ and $\sigma \leq 1$ and this is true because these parameters are probabilities.

Given an initial condition $x_{0} \geq 0$ and a sequence of births $b(0), b(1), \ldots, b(t-1)$, the solution of this system is given by

$$
x(t)=A(\mathbf{p})^{t} x_{0}+\sum_{j=0}^{t-1} A(\mathbf{p})^{t-j-1} B b(j)=A(\mathbf{p})^{t} x_{0}+\sum_{j=0}^{t-1} V(t-j-1, p) b(j) .
$$

where $V(t-j-1, p)=A(\mathbf{p})^{j} B, j \geq 0$ are called the Markov parameters of the systems. It is well-known, [11], that these parameters determine the input/output response/behavior of the system. Then, we can write the input-output response/behavior of the system $(A(\mathbf{p}), B)$ by means of the Markov parameters, which are denoted by $V(j, \mathbf{p}), j \geq 0$.

We recall that, a model is identifiable if and only if there is an unique input-output behavior for every parameter set, that is, if the input-output response of the system is the same for two parameter vectors $\mathbf{p}$ and $\mathbf{q}$, then necessary the parameter vectors are equals, $\mathbf{p}=\mathbf{q}$. There are several techniques to study the identifiability of a system (see for instance [4, 8]). We have chosen to use the Markov parameters to solve this problem. The system (9) is identifiable if and only if, for almost any two candidates parameter vector values $\mathbf{p}$ and $\mathbf{q}, V(j, \mathbf{p})=V(j, \mathbf{q}), j \geq 0$ implies $\mathbf{p}=\mathbf{q}$.

Observe that these Markov parameters have a structure induced by the structure of matrix $A(\mathbf{p})$. Then, the parameter identification process for the structured system is followed from the structure of the vectors obtained by calculation of the Markov parameters of the system. For the system (9) these parameters satisfy that $V_{1}(k, \mathbf{p})=V_{2}(k, \mathbf{p}), V_{3}(k, \mathbf{p})=V_{4}(k, \mathbf{p})$ and $V_{5}(k, \mathbf{p})=V_{6}(k, \mathbf{p})$, where $V_{i}(k, \mathbf{p})$ denotes the $i$-th component of vector $V(k, \mathbf{p})$. Taking into account the last comment from on now this topic is called structurally identifiable, see [15]. From the structure of Markov parameters we solve the identification problem for the system in the next result.

Proposition 2.1 The structured system (9) where $A(\mathbf{p})$ and $B$ are defined by $(10)$ with $\mathbf{p} \in \mathcal{P}$ is structurally identifiable.

Proof. We consider two structured systems defined by $(9)$ with $\mathbf{p}=(c, g, \sigma), \mathbf{q}=(\bar{c}, \bar{g}, \bar{\sigma}) \in \mathcal{P}$ such that they have the same input-output behavior (io)

$$
V(k, \mathbf{p})=V(k, \mathbf{q}), k \geq 0
$$

and we shall prove that $\mathbf{p}=\mathbf{q}$. By the structure of the Markov parameters of system (9) we have

$$
V_{1}(1, \mathbf{p})=V_{1}(1, \mathbf{q}) \Rightarrow c=\bar{c}
$$




$$
\begin{aligned}
& V_{3}(2, \mathbf{p})=V_{3}(2, \mathbf{q}) \Rightarrow g=\bar{g} \\
& V_{3}(3, \mathbf{p})=V_{3}(3, \mathbf{q}) \Rightarrow \sigma=\bar{\sigma}
\end{aligned}
$$

Hence, $\mathbf{p}=\mathbf{q}$ and the system is identifiable.

The last result makes that the parameters $c, g$ and $\sigma$ are uniquely determined by the output structure corresponding to an observation of infection. This result shows that it is appropriate to use the proposed method and output structure to estimate the proportion of immature, susceptible and infected individual in the population.

If we know the input-output behavior of the model given by means of a collection of matrices $V(j, \mathbf{p}), j \geq$ 0 , then the following algorithm allow us determine the parameters $\mathbf{p}$ of the model.

\section{Algorithm}

Step 1 Introduce the matrices $V(j), j=1,2,3$ that determine the known input-output behavior of the model. Introduce the values of $k_{1}, k_{2}$ and $k_{3}$.

Step 2 For $j=1,2,3$ obtain $h=2 j$. Choose the $h$-th row of $V(j)$ and denote it as $v(j)^{h}$.

Step 3 Introduce $k_{0}=p_{0}=0$.

Step 4 Construct the following system

$$
v(j)^{h}=\left(\prod_{i=1}^{j} k_{i-1} p_{i-1}\right)\left(\sum_{i=1}^{j} k_{i}\left(1-p_{i}\right)\right)
$$

Step 5 Solve the above system and obtain the parameters $p_{i}$.

Step $6 c=p_{1}, g=p_{2}, s=p_{3}$. With this process we determinate all the parameters of the system.

Example 2.1 Suppose that the external behavior of a epidemic process during a period of three steps is given by

$$
V(1)=\left(\begin{array}{r}
0.72 \\
0.72 \\
0.08 \\
0.08 \\
0 \\
0
\end{array}\right), V(2)=\left(\begin{array}{l}
0.5184 \\
0.5184 \\
0.0896 \\
0.0896 \\
0.0080 \\
0.0080
\end{array}\right), V(3)=\left(\begin{array}{l}
0.3732 \\
0.3732 \\
0.0776 \\
0.0776 \\
0.0111 \\
0.0111
\end{array}\right) .
$$

We know that $k_{1}=0.8, k_{2}=0.5, k_{3}=0.3$ and we want identify the parameters $c, g$ and $\sigma$. Then we apply the above algorithm:

Step 1 Introduce the matrices $V(1), V(2), V(3)$ and $k_{1}=0.8, k_{2}=0.5, k_{3}=0.3$.

Step 2 Choose the 2-th row of $V(1)$ and denote it as $v(1)^{2}$, the 4-th of $V(2)$ and denote it as $v(2)^{4}$ and the 6-th of $V(3)$ and denote it as $v(3)^{6}$.

Step 3 Introduce $k_{0}=p_{0}=1$. 
Step $4-5$ Construct and solving the following system

$$
\begin{aligned}
& v(1)^{2}=k_{0} p_{0} k_{1}\left(1-p_{1}\right) \\
& v(2)^{4}=k_{0} p_{0} k_{1} p_{1}\left(k_{1}\left(1-p_{1}\right)+k_{2}\left(1-p_{2}\right)\right) \\
& v(3)^{6}=k_{0} p_{0} k_{1} p_{1} k_{2} p_{2}\left(k_{1}\left(1-p_{1}\right)+k_{2}\left(1-p_{2}\right)+k_{3}\left(1-p_{3}\right)\right)
\end{aligned}
$$

and obtain $p_{1}=0.1, p_{2}=0.2, p_{3}=0.1083$.

Step $6 c=0.1, g=0.2, s=0.1083$. With this process we determinate all the parameters of the system.

\section{Disease-free equilibrium points}

In our model we consider that the the birth function can be written as $b(t)=F(t) x(t)$, with $x(t)$ given in (10). Now, the system is given by

$$
x(t+1)=\tilde{A}(\mathbf{p}) x(t)
$$

being $\tilde{A}(\mathbf{p})=A(\mathbf{p})+B F(t)$. The matrix $A(\mathbf{p})$ is transmission matrix and the matrix $B F(t)$ include all other terms (recovery, migration...).

By definition of $g$ we can consider that $g$ is proportional to infectious individuals. Then, $g=\varpi\left(i_{m}(t)+\right.$ $i_{f}(t)$ ), being $\varpi$ the coefficient of proportionality (see [13]).

In this section we use this equation to obtain the equilibrium point, $x^{*}$, when we consider the functions given in (7) and in (8) and we want to find its disease-free equilibrium, that is $i_{m}^{*}=i_{f}^{*}=0$.

\subsection{Linear case}

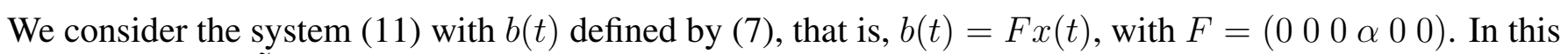
case, the matrix $\tilde{A}(\mathbf{p})$ is given by

$$
\tilde{A}(\mathbf{p})=\left(\begin{array}{cccccc}
k_{1}(1-c) & 0 & 0 & \alpha & 0 & 0 \\
0 & k_{1}(1-c) & 0 & \alpha & 0 & 0 \\
k_{1} c & 0 & k_{2} & 0 & k_{3} \sigma & 0 \\
0 & k_{1} c & 0 & k_{2} & 0 & k_{3} \sigma \\
0 & 0 & 0 & 0 & k_{3}(1-\sigma) & 0 \\
0 & 0 & 0 & 0 & 0 & k_{3}(1-\sigma)
\end{array}\right) .
$$

We want to know if the system has a disease-free equilibrium point. In this point the equation $x^{*}=\tilde{A}(\mathbf{p}) x^{*}$ holds on. That is, we have solve the following system

$$
\begin{aligned}
j_{m}^{*} & =k_{1}(1-c) j_{m}^{*}+\alpha s_{f}^{*} \\
j_{f}^{*} & =k_{1}(1-c) j_{f}^{*}+\alpha s_{f}^{*} \\
s_{m}^{*} & =k_{1} c j_{m}^{*}+k_{2} s_{m}^{*} \\
s_{f}^{*} & =k_{1} c j_{f}^{*}+k_{2} s_{f}^{*},
\end{aligned}
$$


This system has nonzero solution if $|I-\tilde{A}(\mathbf{p})|=0$. This means that $\lambda=1$ is an eigenvalue of the matrix $\tilde{A}(\mathbf{p})$. This condition is true when

$$
\alpha=\left(1+c^{-1}\left(k_{1}^{-1}-1\right)\right)\left(1-k_{2}\right) .
$$

We summarize the above comments in the following result.

Proposition 3.1 If $\alpha$ satisfies $(12)$ the system $(1,6)$ has disease-free equilibrium points $E_{1}(0,0,0,0,0,0)$ and $E_{2}\left(j_{m}^{*}, j\right.$ where

$$
\begin{aligned}
& j_{m}^{*}=j_{f}^{*}=\frac{1-k_{2}}{k_{1} c} s_{f}^{*} \\
& s_{m}^{*}=s_{f}^{*} .
\end{aligned}
$$

The explicit form of the nonzero disease-free equilibrium point is shown to depend on the various model parameters and the modeling assumptions. In particular, it depends on the survival rate of immature and susceptible individuals, the probability of moving from immature to susceptible and the assumption about births.

\subsection{Beverton-Holt type}

In this section we consider the system (1, 6) and we apply the birth function governed by Beverton-Holt type, that is, $b(t)$ is defined by $(8)$. In this case, we obtain the following system

$$
\begin{aligned}
j_{m}^{*} & =k_{1}(1-c) j_{m}^{*}+\frac{\alpha}{1+\beta n^{*}} s_{f}^{*} \\
j_{f}^{*} & =k_{1}(1-c) j_{f}^{*}+\frac{\alpha}{1+\beta n^{*}} s_{f}^{*} \\
s_{m}^{*} & =k_{1} c j_{m}^{*}+k_{2} s_{m}^{*} \\
s_{f}^{*} & =k_{1} c j_{f}^{*}+k_{2} s_{f}^{*} .
\end{aligned}
$$

If the total population in the equilibrium, $n^{*}$, is

$$
n^{*}=\frac{(\alpha-1) k_{1} c-\left(1-k_{1}-k_{2} k\right)}{\beta\left(1-k_{2}\right) k},
$$

where $k=1-k_{1}(1-c)$, the system has nonzero solution. This follows by the same way as in the above subsection. This result is given in the following proposition.

Proposition 3.2 If $n^{*}$ satisfies $(\sqrt{13})$, the system $(1,6)$ has disease-free equilibrium points $E_{1}(0,0,0,0,0,0)$ and $E_{2}\left(j_{m}^{*}\right.$, where

$$
\begin{aligned}
& j_{m}^{*}=j_{f}^{*}=\frac{1-k_{2}}{k_{1} c} s_{f}^{*} \\
& s_{m}^{*}=s_{f}^{*}
\end{aligned}
$$

where $m_{f}^{*}=\frac{n^{*} k_{1} c}{2\left(1-k_{2}+k_{1} c\right)}$.

In this case the nonzero equilibrium point is related to the survival rate of immature and susceptible individuals, the probability of moving from immature to susceptible and the population in the equilibrium. 


\section{Basic reproduction number}

Now, we analyze the stability of the model in the equilibrium. The study is made only for $x^{*}=0$, the stability of any other solution of the model can be studied by changing variables such that the given solution is the zero solution.

The basic reproduction number, denoted as $\mathcal{R}_{0}$, is one the most important parameters in the study of mathematical epidemiology. It is the number of infected individuals in a susceptible population from a single infected case. In discrete-time population models $\mathcal{R}_{0}$ is also referred to as the net reproductive rate(see [1] and the references given there). This value quantifies the transmission potential of a disease and it gives information on transmissibility and contact rates. For that, it can be used to determine if a new infectious disease can invade a population and it can estimate the final epidemic size.

It follows from [12] that when the initial system is asymptotically stable, if $\mathcal{R}_{0}<1$ the disease-free equilibrium is asymptotically stable and if $\mathcal{R}_{0}>1$, it is unstable. If $\mathcal{R}_{0}=1$ the disease becomes endemic, that is the disease remains in the population at a consistent rate.

Consider the system given in (9) with a linear birth function $b(t)=F x(t)$. The matrix $B F$ is known as the fertility matrix and the matrix $A(\mathbf{p})$ as the transition matrix. In this case, the basic reproduction number $\mathcal{R}_{0}$ is given by

$$
\mathcal{R}_{0}=\rho\left(B F(I-A(\mathbf{p}))^{-1}\right),
$$

where matrix $B F(I-A(\mathbf{p}))^{-1}$ is known as the next generation matrix (see [1]) and denoting $\rho(M)$ the spectral radius of a matrix $M$. If the initial system is asymptotically stable, $\rho(A(\mathbf{p}))<1$, and $I_{0}$ is the initial density of infectious individuals, then

$$
B F(I-A(\mathbf{p}))^{-1} I_{0}=B F \sum_{k=0}^{\infty} A(\mathbf{p})^{k} I_{0}
$$

represents the distribution or percentage of all infections accumulated during the lifespan of the population.

So, if $\rho(A(\mathbf{p}))<1$, it follows that $\rho(A(\mathbf{p})+B F)<1$ if and only if $\mathcal{R}_{0}<1$.

Now, we obtain the basic reproduction number in the specific linear case considered in this paper and when we use the Beverton-Holt type control.

\subsection{Linear case}

In this case we consider the matrices $A(\mathbf{p})$ and $B$ given in $(10)$ and the linear control $b(t)$ given in (7). If $\rho(A(\mathbf{p}))<1$, then the reproduction number given in 14 is $\mathcal{R}_{0}=1$, that is the disease remains in the population at a consistent rate, which means that the disease is constantly present in the population.

\subsection{Beverton-Holt type}

Since $b(t)$ is given by (8), the system is a non-linear system and we must linearize the system before to obtain the basic reproduction number.

The linearization of the system around the equilibrium point, $z(t)=x(t)-x^{*}$, gives us the linear system

$$
z(t+1)=(A(\mathbf{p})+B F) z(t)
$$

being $A(\mathbf{p})$ and $B$ the matrices given in 10 and $F=(q q q p q q)$, with $q=-\alpha \frac{\beta s_{f}^{*}}{\left(1+\beta n^{*}\right)^{2}}$ and $p=$ $-\alpha \frac{\beta s_{f}^{*}-\left(1+\beta n^{*}\right)}{\left(1+\beta n^{*}\right)^{2}}$. 
Now, if the initial system is asymptotically stable, we obtain the eigenvalues of the matrix $B F(I-$ $A(\mathbf{p}))^{-1}$ and the basic reproduction number is given by

$$
\mathcal{R}_{0}=\frac{\left(1+(c-1) k_{1}\right)\left(1-k_{2}\right)}{\alpha c k_{1}}
$$

and determines the long-term behavior of the system, when the total population is asymptotically constant. In this case the value of $\mathcal{R}_{0}$ is related with the survival rate of immature and susceptible individual and the probability of immature individual becoming susceptible individual.

Now, we use the Berveton-Holt recruitment function and illustrate the above result in a specific discretetime model. We obtain the basic reproduction number for the disease-free model and analyze the given result. The values of the parameters are chosen for illustration purposes and do not represent actual values.

Example 4.1 Consider the model given in Example 2.1. In this case, from the structural identifiability analysis we have obtained that

Probability of immature individual becoming susceptible individual, $c=0.1$.

Probability that susceptible individual become infectious individual, $g=0.2$.

Probability of infected individual recover, $\sigma=0.1083$.

If we consider $b(t)$ defined as the Beverton-Holt type (8) with $\alpha=3$ and $\beta=0.001$, then we verify that $\rho(A(\mathbf{p}))=0.72<1$, then we can prove that $\rho(A(\mathbf{p})+B F)<1$ if and only if $\mathcal{R}_{0}<1$. For that, we obtain $\rho(A(\mathbf{p})+B F)=0.9278<1$. On the other hand, we obtain $\mathcal{R}_{0}=\frac{\left(1+(c-1) k_{1}\right)\left(1-k_{2}\right)}{\alpha c k_{1}}=0.5833$.

\section{Conclusions}

In this work we use mathematical tools to analyze epidemic processes. A stage-structured model is considered with unknown parameters and the identifiability problem is solved for the model. This fact makes the appropriate model to describe the mechanism of epidemic processes. Equilibrium points are obtained for linear and Beverton-Holt recruitment functions. We compute the reproductive number $\mathcal{R}_{0}$, since the complete identifiability of the parameters allows us determine uniquely the reproductive number. And $\mathcal{R}_{0}$ is used to study the stability of the system. We prove that in the linear case the relationship among the parameters and the stability would not exist. In the Beverton-Holt case the stability is related with the survival rate of susceptible and immature individual and the probability of immature becoming susceptible . Additionally, we give an example to clarify the results.

Acknowledgements. This work has been partially supported by MTM2010-18228.

\section{References}

[1] L.S.J. Allen, P. van den Driessche, The basic reproduction number in some discrete-time epidemic models, Journal of Difference Equations and Applications (2008), pp. 1-19.

[2] L.S.J. Allen, D. B. Thrasher, The effects of vaccination in an age-dependent model for varicella and herpes Zoster number in some discrete-time epidemic models, IEEE Transactions on Automatic Control 43(6) (1998), pp. 779-789. 
[3] A. Ben-Zvi, P.J. McLellan, K.B. McAuley, Identifiability of Linear Time-Invariant DifferentialAlgebraic systems. 2. The Differential-Algebraic Approach, Ind. Eng. Chem. Res. 43(5) (2004), pp. 1251-1259.

[4] C. Boyadjiev and E. Dimitrova, An iterative method for model parameter identification, Computers and Chemical Engineering, 29 (2005), pp. 941-948.

[5] B. Cantó, C. Coll, E. Sánchez, Identifiability of a class of discretized linear partial differential algebraic equations, Math. Problems Eng. (2011), pp. 1-12.

[6] H. Cao, Y. Zhou, The discrete age-strctured SEIT model with application to tuberculosis transmission in China, Mathematical and Computer Modeling (2012), pp. 385-395.

[7] O. Diekmann, J.A.P. Heesterbeek, J.A.J. Metz, On the definition and the computation of the basic reproduction ratio $R_{0}$ in models for infectious diseases in heterogeneus populations, Journal Math. Biol. 28 (1990), pp. 365-382.

[8] J.M. Dion, C. Commault and J. van der Woude, Generic properties and control of linear structured systems: a survey, Automatica, 39 (2003), pp. 1125-1144.

[9] H.E. Emmert, L.S.J. Allen, Population persistence and extinction in a discrete-time, stage-structured epidemic model, Journal of Difference Equations and Applications 10 (2004), pp. 1177-1199.

[10] L. Farina and S. Rinaldi, Positive Linear Systems, John Wiley\&Sons 2000.

[11] T. Kailath, Linear Systems, Prentice-Hall, Englewood Cliffs, N.J. 1980.

[12] C.K. Li, H. Schneider, Applications of Perron-Frobenius theory to population dynamics, Journal Math. Biol. 44 (2002), pp. 450-462.

[13] X. Li, W. Wang, A discrete epidemic model with stage structure, Chaos Solitons and Fractals 26(2005), pp. 947-958.

[14] J. Ma, J.D. Earn, Generality of the final size formula for an epidemic of a newly invading infectious disease, Bull. Math. Biol. 68 (2006), pp. 679-702.

[15] J.M. van den Hof, Structural Identifiability of linear compartmental systems, IEEE Transactions on Automatic Control 43(6) (1998), pp. 800-818.

[16] W. Wang, X. Zhao, An epidemic model in a patchy environment, Math. Biosci. 190 (2004), pp. 97-112. 